\title{
Formación online en fisioterapia. Experiencia de un diseño instruccional de aprendizaje mediante la plataforma virtual Moodle
}

\author{
Rafel Donat-Roca, Violeida Sánchez-Socarrás, Mar Camacho-Martí
}

Introducción. Las características de las tecnologías de la información y la comunicación permiten la realización del life long learning a los profesionales en activo de las ciencias de la salud gracias a la flexibilidad que ofrece la formación online.

Materiales y métodos. Este artículo muestra la experiencia en el uso de la plataforma virtual Moodle dentro de un proyecto de I+D para confeccionar el campus virtual del Col.legi de Fisioterapeutes de Catalunya mediante el curso de formación de voluntariado en cooperación internacional en fisioterapia.

Resultados y conclusiones. La opinión de los fisioterapeutas participantes sobre el uso de la formación virtual es muy favorable. Se destaca el valor que ofrece como espacio de encuentro y crecimiento profesional. Gracias al trabajo colaborativo online se puede construir aprendizaje significativo con una educación sin distancia. La experiencia demuestra que la formación garantiza la adquisición de conocimientos de los fisioterapeutas sin experiencia y la corrección de las deficiencias formativas en los fisioterapeutas con experiencia.

Palabras clave. E-learning. Fisioterapia. Modelos instruccionales. Tecnología educativa. Voluntariado.

Online education in physiotherapy. An experience in the use of instructional learning design with Moodle virtual platform

Introduction. The characteristics of the information and communication technology enable the realization of life long learning to active professionals of the health sciences through the flexibility provided online training.

Materials and methods. This paper shows an experience using Moodle as learning management system which was put into practice in the context of a research and development project whose aim was to design and create a virtual campus of the College of Physiotherapists of Catalonia through volunteer training course on international cooperation in physiotherapy.

Results and conclusions. The opinions of participants physiotherapists provided very favorable towards virtual training. It stands out as a meeting place for professional growth, by means of online collaborative learning significant education can be constructed with no temporal of physical restrictions. Experience has shown that training ensures the acquisition of knowledge by inexperienced physiotherapists and the correction of training deficiencies in the case of experienced physiotherapists.

Key words. Distance learning. Educational technology. Instructional models. Physiotherapy. Volunteerism.

\section{Introducción}

Transformar el modelo de enseñanza clásico, completamente presencial y asumir un nuevo modelo de aprendizaje donde la formación virtual pueda completar e incluso sustituir a la formación tradicional [1] es un reto común a las profesiones sanitarias y las ciencias de la educación para cubrir las demandas de las sociedades del siglo xxi [2]. En este nuevo espacio de enseñanza-aprendizaje es necesario seleccionar los métodos y recursos adecuados para permitir una formación permanente [3].
El campus virtual del Collegi de Fisioterapeutes de Catalunya (CFC) es el resultado de dos años de trabajo de los autores de este artículo para poner en práctica un proyecto de $\mathrm{I}+\mathrm{D}$, con el objetivo de utilizar las tecnologías de la información y la comunicación (TIC) en la adquisición de conocimientos indispensables y en la preservación del life long learning [4], para facilitar la actualización continua de los profesionales de la fisioterapia [5]. La experiencia descrita en este artículo surge de la necesidad de mejorar la formación de los fisioterapeutas de Cataluña, interesados en ejercer como voluntarios en proyec-
Facultad de Ciencias de la Educación y Pedagogía; Universitat Rovira i Virgili; Tarragona (M. CamachoMartí). Escola Universitària de Ciències de la Salut de Manresa, UAB; Departamento de Estudios Universitarios de Fisioterapia; Fundació Universitària del Bages; Manresa, Barcelona, España (R. Donat-Roca, V. Sánchez-Socarrás).

Correspondencia: Prof. Rafel Donat Roca. Escola Universitària de Ciències de la Salut de Manresa (UAB). Departamento de Estudios Universitarios de Fisioterapia. Fundació Universitària del Bages. Avda. Universitària, 4-6. E-08242 Manresa (Barcelona).

Fax: +34938757355.

E-mail: rdonat@fub.edu

Agradecimientos: Al Col-legi de Fisioterapeutes de Catalunya, por optar por la investigación en las tecnologías de la información y la comunicación como forma de preservar la formación constante en fisioterapia.

Conflicto de intereses: No declarado.

Competing interests: None declared.

(c) $2015 \mathrm{FEM}$ 
Tabla I. Aspectos curriculares del cuestionario preformación (Q1). En las siguientes preguntas puedes responder 'no' o 'no lo sé'. En caso de respuesta afirmativa 'si' o 'sí lo sé', justifícala respondiendo correctamente a la pregunta, a partir de tus conocimientos actuales y sin auxiliarte en fuentes de información externa.

1. ¿Sabrías decir en qué consiste la cooperación internacional?

2. ¿Sabes la diferencia entre voluntariado y cooperante?

3. ¿Sabrías decir qué tipo de modelos de cooperación existen en función del momento en que ésta se realiza?

Deficiencia 1 4. ¿Sabrías decir qué tipo de modelos de cooperación existen en función de los participantes?

5. ¿Sabrías definir qué es la anticooperación?

6. ¿Sabrías decir en qué tipo de proyectos de cooperación ha participado históricamente la fisioterapia?

7. ¿Sabrías decir qué es el enfoque del marco lógico de un proyecto de cooperación?

8. ¿Sabrías decir cuál es la patología y la clínica habitual que trata la fisioterapia en los proyectos de cooperación internacional en los que ha participado históricamente?

9. ¿Sabrías decir qué debería saber el fisioterapeuta que quiera participar como profesional en la atención de enfermos tras una catástrofe natural o militar?

10. ¿Sabrías decir para qué podría servir la fisioterapia tras una catástrofe natural como los terremotos acontecidos en Chile y Haití?

Deficiencia 2 11. ¿Sabrías decir qué debería saber el fisioterapeuta que quiera participar como profesional en la atención de enfermos en una situación de conflicto bélico?

12. ¿Sabrías decir en qué consiste la capacitación técnica para el desarrollo?

13. ¿Sabrías decir qué características tiene la discapacidad intelectual infantil en los países en vías de desarrollo?

14. ¿Sabrías decir cuáles son las prioridades de la fisioterapia en el abordaje de la discapacidad intelectual infantil en los países en vías de desarrollo?

tos de cooperación internacional. Esta necesidad se convirtió en una motivación para realizar una prueba piloto del sistema instruccional seleccionado en modalidad e-learning para la formación online.

Hasta el 2010, año en que se inició esta investigación, ningún fisioterapeuta había recibido formación virtual en cooperación internacional en España. Las formaciones presenciales o semipresenciales que preceden a este estudio no son específicas para la fisioterapia, sino que están incluidas en programas de ciencias de la salud dirigidas principalmente a médicos y a enfermeras. En una investigación anterior [6], además de confirmar estos datos, se obtuvo que los fisioterapeutas valoraron su formación previa como inexistente o con importantes deficiencias en temas relacionados con las características propias de un voluntariado, los conocimientos técnicos de los diferentes proyectos de coopera- ción internacional existentes y la capacitación para afrontar con eficacia el proceso de atención clínica de la población beneficiaria directa de la fisioterapia. Ante esta realidad se planteó la necesidad de crear un curso formativo acreditado por el Consell Català de Formació Continuada de les Professions Sanitàries -comisión de formación continuada del Sistema Nacional de Salud-, diseñado con el objetivo de brindar a los fisioterapeutas interesados en participar en proyectos de cooperación internacional los conocimientos necesarios a través de una formación flexible [7] y de una docencia adaptada a las directrices de la declaración de Bolonia, que aboga por facilitar la personalización y tutorización de la enseñanza universitaria adaptada a la enseñanza continuada profesional $[8,9]$.

El objetivo general de este artículo es evaluar la utilidad de la modalidad e-learning para la formación profesional en fisioterapia en el ámbito de actuación de la cooperación internacional. Como objetivos específicos se pretende:

- Evaluar el nivel de conocimiento previo en cooperación internacional de los fisioterapeutas participantes en el estudio.

- Valorar la utilidad de la modalidad e-learning para mejorar el nivel de conocimiento previo.

- Identificar y valorar aspectos cualitativos de la metodología e-learning utilizada entre los grupos de intervención respecto al grupo control.

- Recoger las propuestas de mejora de todos los grupos participantes en la intervención.

\section{Materiales y métodos}

\section{Descripción del método implementado}

Para esta formación se definieron las siguientes competencias específicas:

- Conocer los requisitos que debe cumplir un fisioterapeuta para realizar un voluntariado en cooperación internacional y que permiten diferenciarlo del turismo solidario y de la cooperación profesional.

- Ser capaces de identificar los diferentes tipos de proyectos de cooperación y sus requerimientos profesionales.

- Ser capaces de identificar, planificar y desarrollar la intervención profesional en las afecciones clínicas más comunes que competen a los fisioterapeutas durante la cooperación internacional.

- Conocer la definición y características del enfoque del marco lógico de un proyecto de cooperación internacional. 
La distribución de los contenidos y las actividades de la formación online se organizaron de la siguiente manera:

\section{Sesión informativa presencial}

En una sesión informativa inaugural en calidad de presentación del curso, los fisioterapeutas recibieron una explicación de la dinámica de funcionamiento del campus, de cómo las metodologías propuestas pretenden convertir al alumno en el centro del aprendizaje [10], y del grado de implicación que conlleva la formación virtual. Esta sesión, en la que participó el $80 \%$ de los alumnos matriculados, fue el único contacto presencial entre profesor y alumnos, con el objetivo de potenciar una proximidad facilitadora de la relación virtual durante la formación [11]. La sesión fue grabada y colgada en la cabecera del aula virtual mediante el programa Vimeo para permitir su reproducción.

En la sesión inaugural participaron además dos miembros del departamento de formación del CFC, adiestrados previamente en la plataforma virtual y en el sistema instruccional del curso para solucionar dificultades técnicas, supervisar y mediar en la comunicación entre profesores y alumnos y guiar a ambos a través del cronograma formativo.

\section{Aula virtual y sistema instruccional}

Se diseñaron cuatro bloques temáticos con una organización interna que favorece la proximidad, la interacción, la construcción colectiva del conocimiento y la comprobación del aprendizaje individual [12]. Cada bloque se inició con un video del profesor explicando los contenidos y las actividades a realizar. A continuación se relacionaron los materiales didácticos, las actividades de aprendizaje individual para profundizar en los contenidos seleccionados y las actividades de aprendizaje colaborativo para debatir, contrastar y verificar lo aprendido, y construir así un nuevo aprendizaje colectivo.

\section{Materiales}

Incluyeron contenidos seleccionados por las diferentes comisiones profesionales del CFC presentados en diferentes formatos (pdf, slideshare, documentos audiovisuales o enlaces externos), en función del contenido y de la necesidad de aprendizaje, pensados para garantizar la interacción del alumno con un material didáctico agradable y motivador, sin perder la rigurosidad del contenido.

\section{Trabajo individual}

Se utilizaron dos tipos de actividades de Moodle: las lecciones y los entregables. Las lecciones se uti-
Tabla II. Aspectos curriculares del cuestionario posformación (Q2).

1. Define en un máximo de cinco líneas en qué consiste la cooperación internacional

2. Explica con ejemplos qué requisitos debería cumplir un fisioterapeuta que quisiera entrar en la cooperación internacional como cooperante, como voluntario o como brigadista en un viaje solidario

3. Explica qué tipo de modelos de cooperación existen en función del momento en que ésta se realiza. Aporta un ejemplo para cada modelo para reforzar tu contestación

Deficiencia 14 4. Explica qué tipos de modelos de cooperación existen en función de los participantes. Aporta un ejemplo de cada modelo para reforzar tu contestación

5. Explica con un ejemplo cómo puede la fisioterapia caer en la anticooperación

6. Define en qué tipo de proyectos de cooperación ha participado históricamente la fisioterapia. Cita un ejemplo para apoyar tu contestación.

7. Explica por qué un fisioterapeuta que quiera realizar un proyecto de cooperación internacional debe conocer qué es el enfoque del marco lógico. Pon un ejemplo para reforzar tu contestación

8. Explica cuál es la patología y la clínica habitual que atiende la fisioterapia en los proyectos de cooperación internacional en los que ha participado históricamente

9. Explica qué debería saber el fisioterapeuta que quiera participar como profesional en la atención de enfermos tras una catástrofe natural o militar

10. Cita cuáles serían los objetivos de la fisioterapia tras una catástrofe natural como los terremotos acontecidos en Chile y Haití

Deficiencia 2 11. Cita cuáles serían los objetivos de la fisioterapia tras o durante un conflicto bélico

12. Explica en qué consiste la capacitación técnica para el desarrollo. Aporta un ejemplo relacionado con la fisioterapia que permita reforzar tu contestación

13. Explica qué características tiene la discapacidad intelectual infantil en los países en vías de desarrollo

14. Cita cuáles son los objetivos de la fisioterapia en el abordaje de la discapacidad infantil en los países en vías de desarrollo

lizaron con la finalidad de reforzar el estudio del material didáctico e incluyeron preguntas abiertas (a corregir por el profesor) y preguntas cerradas (de autoevaluación del alumno), aprovechando la flexibilidad para el autoaprendizaje que ofrecen las TIC [13]. La finalidad de los entregables fue potenciar la abstracción de lo esencial de cada módulo, a través de la entrega de un documento resumen en formato Word o pdf, a corregir por el profesor y que garantizaría la orientación al alumno con un feedback continuo y sistemático [14].

\section{Trabajo colaborativo}

Las actividades de Moodle utilizadas fueron los foros, las wikis y los glosarios. Los estudiantes pudieron disponer de espacios colaborativos de aprendi- 
zaje donde abocar tanto las dudas como las conclusiones una vez comprobado el grado de conocimiento individual. Estas herramientas permiten que los alumnos aprendan a compartir y a construir colectivamente un aprendizaje homogéneo y activo [15]. En los espacios colaborativos los alumnos contaron con el seguimiento y tutorización del profesor, para mantener la continuidad en el sistema de enseñanza-aprendizaje [16].

\section{Sistema de mensajería}

El sistema de mensajería de la plataforma Moodle se sistematizó en función del dominio de la herramienta por parte de los alumnos y de los requisitos de la actividad de aprendizaje. Se utilizó el correo electrónico para concertar las tutorías virtuales realizadas mediante Skype, resolver las dudas individuales, recordar las fechas de entrega y esclarecer dudas sobre la dinámica general del curso. En las lecciones, los cuestionarios y los entregables, se utilizó la opción propia de estas herramientas de Moodle para garantizar el feedback con el profesor, mientras que las wikis, glosarios y foros, por tratarse de objetos de aprendizaje virtual que permiten utilizar la participación en abierto con el profesor y entre los alumnos, fueron la opción utilizada para resolver las dudas inherentes al trabajo colaborativo.

\section{Calificación final}

La calificación final se determinó a partir de las siguientes proporciones: actividades individuales, $20 \%$; participación en los foros de debate y opinión, $10 \%$; participación en la construcción colaborativa a través de wikis y glosarios, $10 \%$, y resolución del cuestionario final (Q2) sobre los aspectos curriculares de la cooperación internacional, $60 \%$.

\section{Evaluación cuantitativa del aprendizaje mediante cuestionarios}

Se realizó un estudio cuasiexperimental por comparación interna entre dos grupos experimentales de intervención (GE1, sin experiencia previa, y GE2, con experiencia previa) y comparación externa entre los grupos de intervención y un grupo control que no realizó la formación e integrado por fisioterapeutas con experiencia previa en al menos un voluntariado en los últimos 15 años, seleccionados aleatoriamente entre los censados en el CFC. El departamento de formación del CFC eligió a los integrantes de los grupos GE1 y GE2 por estricto orden de matriculación al curso.

Se administraron dos cuestionarios semiestructurados: preformación (Q1) (Tabla I) y posforma- ción (Q2) (Tabla II). Ambos cuestionarios incluyeron preguntas cerradas sobre el perfil de los profesionales y preguntas abiertas sobre el grado de conocimientos en relación a los aspectos curriculares de la cooperación internacional. Las 14 preguntas de los aspectos curriculares se agruparon en dos apartados denominados 'deficiencias'. La deficiencia 1 corresponde a los conocimientos básicos sobre los conceptos técnicos y definiciones de la cooperación internacional, y la deficiencia 2, a los conocimientos necesarios sobre el proceso de atención clínica en las patologías más comunes, atendidas en los proyectos de cooperación internacional.

La participación del grupo control en este tramo de la investigación consistió en la realización del cuestionario Q2 en el mismo período que los grupos de intervención, permitiendo la comparación externa de los resultados cuantitativos.

\section{Evaluación cualitativa del aprendizaje mediante grupos focales}

Al finalizar la formación, y una vez que los integrantes del grupo control tuvieron la oportunidad de visualizar el contenido y el material didáctico del curso y de probar el funcionamiento del aula virtual, se constituyeron tres grupos focales para conocer la valoración cualitativa de la propuesta educativa online [17]. Participaron 27 fisioterapeutas (nueve de cada grupo), seleccionados de forma aleatoria por el departamento de formación del CFC.

Los cuestionarios Q1, Q2 y las preguntas a formular en los grupos focales fueron elaborados por el grupo de trabajo de cooperación internacional del CFC y validados por consenso entre expertos en tecnología educativa independientes a la investigación y miembros del Departamento de Pedagogía de la Universitat Rovira i Virgili. Todos los participantes firmaron un consentimiento informado que fue registrado y archivado por el mencionado departamento del CFC y que garantizó su participación voluntaria en un proyecto de investigación.

\section{Resultados}

La investigación incluyó un total de 100 fisioterapeutas, 41 constituyeron el GE1 y 19 el GE2. El $88,33 \%(n=53)$ finalizaron la formación y sólo siete abandonaron el curso por razones familiares o laborales (cinco del GE1 y dos del GE2). A pesar de la predisposición inicial, estos alumnos vieron reducida su disponibilidad para ejecutar las tareas formativas a las que se habían comprometido inicialmen- 
te. De los 40 fisioterapeutas del grupo control, 35 contestaron y finalizaron el cuestionario Q2 en el tiempo establecido de dos semanas.

\section{Valoración cuantitativa del sistema de aprendizaje}

Los resultados obtenidos de la aplicación del cuestionario Q1 a los grupos de intervención preformación se muestran en la figura 1 y revelan el desconocimiento de los fisioterapeutas en temas de cooperación internacional, independientemente de su experiencia previa como voluntarios.

Los resultados de la aplicación del cuestionario Q2 se muestran en la figura 2 y describen una notable mejora de los resultados de aprendizaje posformación de los grupos de intervención independientemente de la experiencia previa como voluntarios en cooperación internacional. En ambas deficiencias se obtiene una mejora significativa de casi 5 puntos sobre 10 posibles, resultados que se ajustan al rango de normalidad situado entre 6 y 8 puntos sobre 10, según los expertos.

La calificación media de los dos grupos de intervención fue de 7,5 para el GE1 (fisioterapeutas sin experiencia previa) y 7,8 para el GE2 (fisioterapeutas con experiencia previa).

Los resultados del grupo control, por el contrario, confirman las deficiencias formativas a pesar de la experiencia, deficiencias reveladas por una nota media entre ambas deficiencias de 3,25, muy por debajo de la obtenida por los grupos de intervención.

\section{Valoración cualitativa del sistema de aprendizaje}

\section{Opinión del grupo control}

- Necesidad de incluir otros complementos en la utilización del campus: 'En general todo es muy intuitivo y el tutorial de bienvenida te facilita mucho las cosas, pero yo añadiría algún tutorial más.

- Dificultad en el seguimiento de los foros de debate $y$ de interacción en la wiki: 'Creo que en los foros de debate los grupos no deberían ser de más de seis miembros; si no, será difícil poder seguir la discusión y guiar el aprendizaje'. 'La wiki parece menos interactiva que el foro; si no hay debate sobre lo que se cuelga, deberá especificarse muy bien su finalidad para que sea más interesante'.

- Actualidad y profundidad del material didáctico que además fue presentado de forma atractiva: 'Me parece muy interesante todo el contenido expuesto, de hecho me doy cuenta de que hay cosas que no sé y debería saber.' 'Creo que está todo, que no falta nada y que la forma tan plural
Figura 1. Resultados de los grupos de intervención en el cuestionario Q1 previo a la formación.

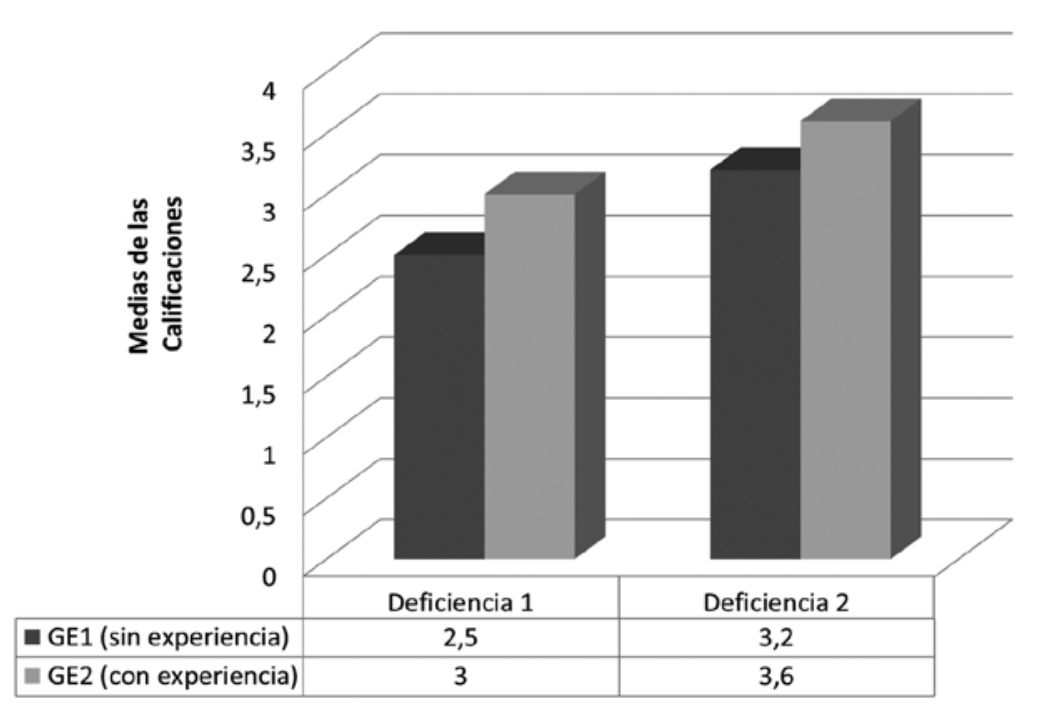

Figura 2. Resultados de la aplicación del cuestionario Q2 en los grupos de intervención (GE1 y GE2) al finalizar su formación, en relación con el grupo control (GC).

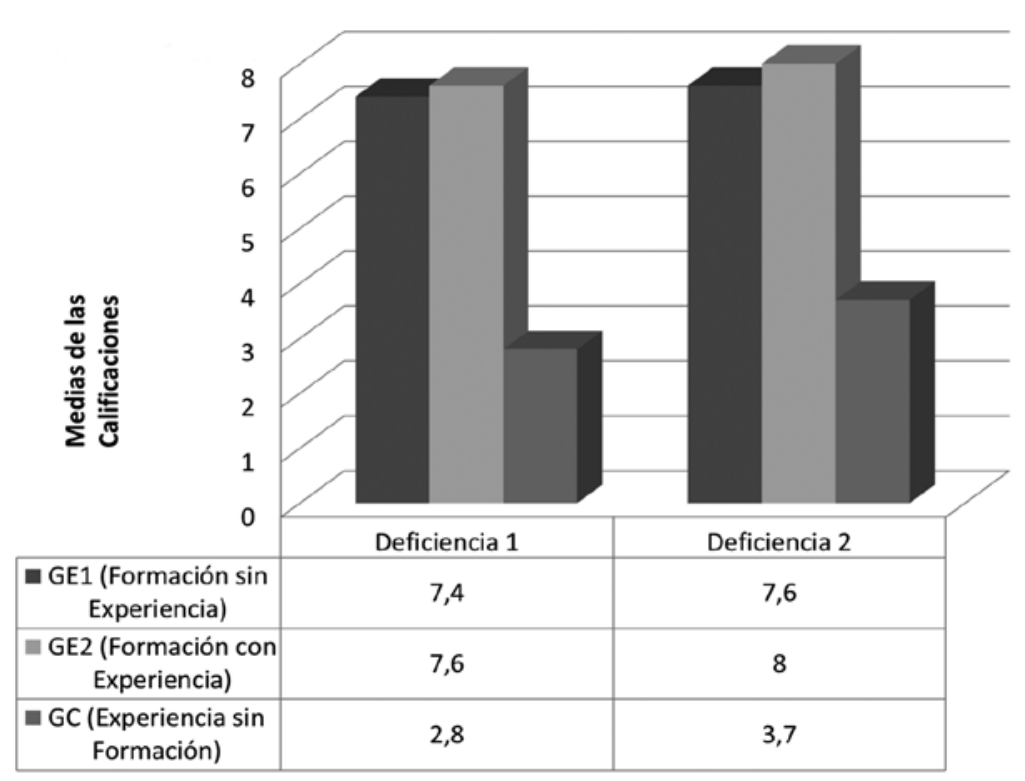

de presentarlo con imágenes, videos y enlaces de apoyo lo hace aún más atractivo si cabe'.

- Facilidad en el seguimiento del aprendizaje: la cantidad de herramientas para la libre comuni- 
cación entre los alumnos y entre éstos y el profesor se considera una garantía en el control y seguimiento del aprendizaje, necesarios en la formación online para evitar el distanciamiento y el abandono de la formación: 'Mucho trabajo para el profesor pero ideal para el alumno.' 'Es una cuestión cultural pero el hecho de ver la cara a tu profesor mediante un video en cada módulo desde un punto de vista antropológico facilita mantenerte enganchado a la formación.' 'El feedback constante le da un plus por encima de la formación presencial y eso beneficia al alumno'.

- Ausencia de formación referente: 'En mi caso hubiera sido interesante hacerlo antes de realizar mi voluntariado, hubiese aprovechado mucho más mi experiencia.' 'Esta formación te permite hacer un voluntariado más reflexionado y maduro por tener más conocimientos previos útiles'.

\section{Opinión de los grupos de intervención}

Sobre las fortalezas del método. Se destacó la flexibilidad del trabajo a través de la plataforma virtual que permitió adaptar el curso a la progresión del aprendizaje y a la disponibilidad de acceso por parte del alumno, sin obligar al alumno a adaptarse al curso: 'Necesitaba continuar aprendiendo y que la formación se adaptase al tiempo que tengo para aprender.' 'Una experiencia muy recomendable si no tienes tiempo y vives lejos de donde se hace el curso'.

También se valoraron positivamente los contenidos y el formato en que éstos fueron presentados: 'He aprendido mucho por la diversidad de los materiales, motiva formarse así. 'El contenido es actual y el sistema, motivante.' 'Si todo fueran pdfs sería muy aburrido.' 'Que algunos documentos se pudieran imprimir era cómodo por no tener que estar siempre conectado'.

Finalmente se destacó la formación a distancia como un espacio de encuentro para unificar criterios entre profesionales que comparten un ámbito de actuación o inquietudes similares al respecto: 'No me esperaba compartir tanto desde un ordenador, una experiencia muy positiva que engancha'. 'Aprendes no sólo de tu trabajo, sino también con el de tus compañeros.' 'Nos permite debatir e intercambiar impresiones desde distintas experiencias para unificar criterios'.

Puntos débiles. Los integrantes de los grupos de intervención manifestaron errores técnicos en algunas actividades de autoaprendizaje, básicamente en las lecciones: 'Alguna vez, si la respuesta era correcta, no te dejaba avanzar en la lección'. 'A veces dis- ponías de dos oportunidades para responder a las preguntas, y al hacerlo, resultaba que tenías más'.

También hicieron una reflexión respecto al cumplimiento del calendario del curso: 'Si bien se agradece la flexibilidad, en las actividades grupales a veces sería mejor una fecha de entrega no tan amplia.' 'El hecho de no cerrar los bloques ya realizados permitía poder rezagarte en algunos momentos y esto a veces era una dificultad para seguir el ritmo de los demás'

Propuestas de mejora. Las propuestas de mejora de los fisioterapeutas de los grupos de intervención se centraron en la temporalización o carga de información en los espacios colaborativos de aprendizaje: 'A veces, cuando querías intervenir, tenías la sensación de que ya se había dicho todo, sería recomendable hacer grupos más pequeños en algunas actividades'. 'Si el espacio sólo es para colgar ejemplos y no hay interacción como en la wiki, aunque es interesante, las aportaciones pierden interés.

\section{Discusión}

El análisis de los resultados revela dos aspectos positivos a destacar: el escaso grado de abandono de la formación (sólo un 11,67\%) y las excelentes calificaciones y opiniones obtenidas por los integrantes de los grupos de intervención en el cuestionario posformación y en los grupos focales. Los fisioterapeutas son profesionales de la salud con horarios laborales muy variables, en puestos de trabajo localizados razonablemente distantes de las instituciones formativas y obligados a combinar la formación constante con otras actividades laborales y sociales; sin embargo, su disponibilidad para formarse y la flexibilidad que ofrece la formación virtual se convierten en un factor clave que justifica las calificaciones obtenidas [18]. Esto coincide con otros estudios donde se muestra que la plataforma virtual permite el acercamiento y adaptación a las nuevas demandas profesionales [19-21].

Para analizar los resultados de los cuestionarios es necesario un diseño instruccional que permita relacionar los buenos resultados con una metodología que garantice el aprendizaje [22]. Una de las técnicas más efectivas para mejorar los cursos de formación es la evaluación continúa con feedback constante a los estudiantes [23]. La amplia diversidad de herramientas síncronas (videoconferencia) o asíncronas (correo electrónico, actividades de autoaprendizaje y foros) utilizadas en el curso para la interacción entre los alumnos y entre éstos y el pro- 
fesor se plantean como una de las claves fundamentales que explican los beneficios del rol de guía del profesor y el rol activo-participativo del estudiante hacia su aprendizaje [24].

La disposición de la trayectoria individual de aprendizaje a continuación del video explicativo o tutorial online coincide con las ventajas manifestadas en estudios anteriores [12] sobre la utilización de programas informáticos educativos que permitan asegurar una interacción del alumno con los materiales escogidos por los expertos en cada una de las temáticas seleccionadas. Para conseguir que el aprendizaje sea significativo, compartido y debatido con los demás, es imprescindible tener un conocimiento previo de la temática a tratar. Este conocimiento debe regularse mediante un feedback inmediato dentro del objeto de aprendizaje seleccionado. En este caso fueron preferentemente las lecciones de Moodle, por su flexibilidad en la construcción, por la facilidad de conexión con el material didáctico referente y por las diferentes opciones de feedback que ofrecen [25].

La disposición de la trayectoria colaborativa de aprendizaje en la parte final de cada bloque permite construir colectivamente un muro de información compartida. El hecho de que el alumno se sienta parte activa y responsable de la construcción del conocimiento enriquece la motivación y anima a la participación con la aportación libre de enlaces externos que puedan ilustrar o ejemplificar los contenidos expuestos. Durante el curso de formación se confirmó la importancia del uso de foros en la zona de trabajo para compartir los conocimientos adquiridos y resolver las dudas [26,27]. Se incentivó la participación activa de los alumnos proponiendo soluciones, planteando y resolviendo dudas. Su uso exhaustivo lo convierte en la mejor herramienta de tutorización y aprendizaje colaborativo, donde los alumnos pueden ser parte activa de la construcción de la enseñanza-aprendizaje. Esta utilización de los foros complementa los tutoriales individuales que significan las lecciones, pero en este caso en grupo y de forma colaborativa, para controlar el avance del alumno sobre el material multimedia y ofrecer una amplia gama de caminos y direcciones para que éste construya su aprendizaje [28]. En estos espacios se demostró la importancia de la participación compartida entre alumnos y docentes [29]. En ellos, el profesor puede proponer trabajos y los alumnos pueden dejar sus dudas y, a la vez, corregir las dudas de otros compañeros, en un entorno de aprendizaje colaborativo supervisado por el profesor, que en nuestra opinión no debe intervenir a menos que resulte evidente que los alumnos no son capaces por sí solos de encontrar la solución y resulte necesaria alguna pista adicional. Estas herramientas, junto con el glosario y la wiki [30], conformaron un depósito de conocimiento que ayudó a dinamizar el desarrollo del curso.

Los datos obtenidos confirman la eficacia del sistema de enseñanza-aprendizaje y la significación de la formación online para corregir las deficiencias formativas y para la adquisición de nuevos conocimientos. Las claves de la significación de la propuesta en la modalidad de e-learning dentro del proyecto de I+D pasan por la actualidad del contenido, la modernización en la presentación de los materiales, la proximidad y la interacción constante del profesor en el aprendizaje del alumno, y la libertad y contribución activa al resultado final de la formación por parte de los estudiantes. Estos aspectos formativos transversales a cualquier ámbito de actuación hacen pensar que es factible su aplicación como sistema instruccional en otras áreas clínicas o profesionales de la fisioterapia.

\section{Bibliografía}

1. Marqués P. Les TIC a l'educació social: entorns de treball i exemples d'ús. Revista Quaderns d'Educació Social 2008; 12: 159-73.

2. Baelo R. El e-learning, una respuesta educativa a las demandas de las sociedades del siglo xxI. Pixel-Bit, Revista de Medios y Educación 2009; 35: 87-96.

3. Mellado E, Talavera MC, Romera F, García MT. Las TIC como herramienta fundamental de la formación permanente en la Universidad de Sevilla. Pixel-Bit, Revista de Medios y Educación 2011; 39: 155-66.

4. Lain D, Aston J. Literature review of evidence on e-learning in the workplace. London: Institute of Employment Studies; 2004.

5. Peacock S, Hooper J. E-learning in physiotherapy education. Physiotherapy 2007; 93: 218-28.

6. Donat-Roca R, Camacho-Martí M. Estudio descriptivo sobre el estado formativo del voluntariado de fisioterapia en cooperación internacional. Perspectiva del uso de las tecnologías de la información y la comunicación. Fisioterapia 2013; 35: 197-205.

7. Cebrián de la Serna M, Ríos JM. Nuevas tecnologías aplicadas a las didácticas especiales. Madrid: Pirámide; 2000.

8. González JC. TIC y la transformación de la práctica educativa en el contexto de las sociedades del conocimiento. Revista de Universidad y Sociedad del Conocimiento UOC 2008. URL: http://www.uoc.edu/rusc/5/2/dt/esp/gonzalez.pdf. [15.01.2013].

9. García C. Nuevas tecnologías y educación. Revista Telos 1991; 28: 9-10.

10. Salinas J, Pérez A, De Benito B. Metodologías centradas en el alumno para el aprendizaje de la red. Madrid: Síntesis; 2008.

11. Chiecher A, Donolo D, Rinaudo MC. Manejo del tiempo y el ambiente en una experiencia didáctica con instancias presenciales y virtuales. RED, Revista de Educación a Distancia 2008. URL: http://www.um.es/ead/red/20/chiecher.pdf. [10.02.2013]

12. Urbina S. Informáticas y teorías del aprendizaje. Pixel-Bit, Revista de Medios y Educación 1999. URL: http://www.sav. us.es/pixelbit/pixelbit/articulos/n12/n12art/art128.htm. [10.02.2013].

13. Cabero J, Romero R. Diseño y producción de TIC para la formación. Barcelona: UOC; 2007. 
14. Sánchez A, Boix JL, Jurado P. La sociedad del conocimiento y las TIC: una inmejorable oportunidad para el cambio. Pixel-Bit, Revista de Medios y Educación 2009; 34: 179-205.

15. Zea C. Un ambiente de aprendizaje colaborativo basado en tecnologías de información y comunicaciones. Revista Sistemas 1996; 68.

16. Marcelo C. La función docente: nuevas demandas en tiempos de cambio. Madrid: Síntesis; 2001.

17. Kreuger R, Casey M. Focus groups: a practical guide for applied research. Thousand Oaks, CA: Sage Publications; 2000.

18. Cook D, Levinson A, Garside S, Dupras D, Erwin P, Montori V. Internet based learning in the health professions: a metaanalysis. JAMA 2008; 300: 1181-96.

19. Quero A, Guillamet A, Pérez F, González P, Garach J, Villanueva C. Valoración cualitativa del alumnado sobre el aprendizaje basado en problemas. XII Encuentro de Investigación en Enfermería y $6{ }^{\circ}$ Coloquio Bianual Joanna Briggs. Madrid: Instituto de Salud Carlos III/Unidad de Coordinación y Desarrollo de la Investigación en Enfermería 2008. p. 397-8.

20. López-Morales M, Celma-Vicente M, Cano-Caballero Gálvez MD, Quero-Rufián A, Rodríguez-López MA. Docencia universitaria semipresencial. Experiencia en el uso de la plataforma virtual SWAD. Educ Med 2011; 14: 229-34.

21. Mora A, Mérida E. Metnum: una experiencia de utilización de nuevas tecnologías y herramientas, para la mejora en los procesos de aprendizaje. Pixel-Bit, Revista de Medios y Educación 2010; 38: 201-14.
22. Anguera MT, Chacón S, Blanco V. Evaluación de programas sociales y sanitarios: un abordaje metodológico. Madrid: Síntesis; 2008.

23. Pina A. Blended learning. Conceptos básicos. Pixel-Bit, Revista de Medios y Educación 2004; 23: 7-20.

24. Cubo S, González JJ, Lucero M. Perspectiva pedagógica de los multimedia. Revista Española de Pedagogía 2003; 225 : 309-35.

25. Paragina F, Paragina S, Jipa A, Savu T, Dumitrescu A. The benefits of using Moodle in teacher training in Romania. Procedia, Social and Behavioral Sciences 2011; 15: 1135-9.

26. Vivina R. El foro electrónico: una herramienta tecnológica para facilitar el aprendizaje colaborativo. Edutec, Revista Electrónica de Tecnología 2004. URL: http://edutec.rediris. es/Revelec2/Revelec17/brito_16a.htm. [03.03.2013].

27. Cataldi Z, Cabero J. Los aportes de la tecnología informática grupal interactivo: la resolución de problemas a través de foro de discusión y de chat. Pixel-Bit, Revista de Medios y Educación 2006; 27: 115-30.

28. Bravo JL. Medios de enseñanza: clasificación, selección y aplicación. Pixel-Bit, Revista de Medios y Educación 2004; 24: 113-24.

29. Bartolomé A. El profesor cibernauta. Barcelona: Graó; 2008.

30. Del Moral ME, Villalustre L. Las wikis vertebradoras del trabajo colaborativo universitario a través de Web Quest. Revista Latinoamericana de Tecnología Educativa 2008; 7: 73-83. 